\title{
Clinical Trials, Trauma, and Neuropsychiatric Comorbidity
}

\author{
By Eric Hollander, MD
}

Welcome back from summer vacation. I hope that you are refreshed and ready for another academic year.

This month's CNS Spectrums introduces new clinical trials data in generalized anxiety disorder and pediatric bipolar disorder, discusses exposure therapy and hypothalamic-pituitaryadrenal dysfunction associated with trauma, and presents an unusual case of neurologic/ psychiatric comorbidity.

Pediatric bipolar disorder has been the focus of increasing research interest and presents with unique features, such as irritability and lack of euphoria, in contrast to adult bipolar disorder. There is also evidence that treatment response in pediatric bipolar disorder may differ from that seen in adult bipolar disorder. Joseph Biederman, MD, and colleagues conducted an open-label, prospective study of aripiprazole monotherapy in pediatric bipolar disorder. Since open-label treatment was beneficial, placebo-controlled, double blind studies are warranted to fully study efficacy and tolerability issues in this unique population.

It has been observed that the psychic and somatic symptoms of generalized anxiety disorder do not respond uniformly in the first weeks of treatment. Stephen M. Stahl, MD, $\mathrm{PhD}$, and colleagues conducted a post-hoc analysis to describe the temporal response to treatment for specific Hamilton Rating Scale for Anxiety items during treatment with venlafaxine extended release. The earliest symptoms to respond included both psychic symptoms (anxious mood, tension, behavior at interview) and somatic muscular, cardiovascular, and respiratory symptoms. The last symptoms to respond included the psychic symptoms of insomnia and fear and the somatic sensory, gastrointestinal and autonomic symptoms. Continuing treatment beyond 8 weeks in venlafaxine extended release responders may improve late-responding symptoms, perhaps in part by the development of tolerance to antidepressant side effects.

Civilian and combat posttraumatic stress disorder (PTSD) may differ in several respects, including response to treatment. Prolonged exposure therapy found efficient in reducing PTSD symptoms among rape victims has not been explored to date in combat-related PTSD. Nitsa Nacasch, MD, and colleagues describe five patients with severe chronic PTSD, unresponsive to previous treatment (medication and supportive therapy), who received $10-15$ sessions of prolonged exposure therapy. All five patients showed marked improvement, with four maintaining gains for up to 18 months.

Early lifetime trauma, abuse, and neglect may have powerful epigenetic effects, resulting in long-term symptoms expressed across various disorders. While blunted and enhanced cortisol suppression following a dexamethasone test are described in eating disorders, some evidence suggests that enhanced cortisol suppression may be associated with the presence of trauma history. Marina Dlaz-Marsá, MD, and colleagues studied 52 patients with eating disorders and found patients with bulimia symptoms had greater cortisol suppression than controls and anorexia patients, and that cortisol suppression was significantly correlated with intensity of childhood traumatic events. This may reflect early gene $x$ environment interactions.

Dr. Hollander is the editor of this journal, Esther and Joseph Klingenstein Professor and Chairman of Psychiatry at the Mount Sinai School of Medicine, and director of the Seaver and New York Autism Center of Excellence in New York City. 
Finally, Mark A. Walterfang MBBS (Hons), FRANZCP, and colleagues describe adrenomyeloneuropathy (AMN), which has to date been associated with only occasional cases of major mental illness. Given that cerebral involvement occurs in up to half of AMN sufferers, they examined whether rates of mental illness may be higher than previously believed. Ten genetically confirmed AMN sufferers underwent structured diagnostic psychiatric interviews. While generally cognitively intact, there was a higher than expected prevalence of lifetime and current major affective illness. Psychopathology did not relate to adrenal status nor to level of physical or functional impairment. Thus, AMN may not be a "pure" myeloneuropathy, and mild cerebral involvement may be associated with affective illness.

Clearly, there is a need for clinical trials to disentangle time course of symptom response in complex disorders, as well as pediatric variants of what is usually considered adult illnesses. Trauma, unfortunately, is becoming a greater and greater part of our modern world, and a better understanding of the epigenetic effects of early trauma, and the development of new treatments for both combat and civilian trauma are needed. Finally, even neurologic disorders that are not frequently thought to be complicated by psychiatric comorbidity need to be carefully examined for such, since this may influence long-term course of illness and treatment response. CNS

\section{MICHAEL E. THASE, MD \\ JOINS CNS SPECTRUMS' EDITORIAL ADVISORY BOARD}

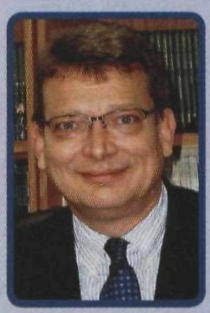

Dr. Thase is Professor of Psychiatry at the University of PennsylvaniaSchoolofMedicine and is a Distinguished Fellow of the American Psychiatric Association, a Founding Fellow of the Academy of Cognitive Therapy, and has been elected to the membership of the American College of Psychiatrists and the American College of Neuropsychopharmacology.

"Dr. Thase is an internationally renowned clinical investigator and runs one of the largest programs in mood disorders, with a primary focus in the evaluation of pharmacologic and psychotherapeutic strategies for unipolar and bipolar depressions," said Eric Hollander, MD, Editor in Chief of CNS Spectrums.

"We are very pleased to we come Dr. Thase to the board and look forward to his immediate contribution to the journal," added Darren L. Brodeur, CEO \& Publisher.

CNS Spectrums is the largest, peerreviewed, scientifically indexed neuropsychiatric journal in the U.S., reaching more than 50,000 physicians monthly. 


\section{NEI Psychopharmacology ACADEMY 2-Day Series}

a

NEI

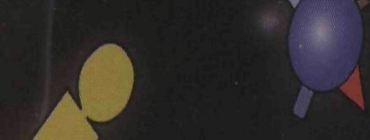

Are you an

NEl member?

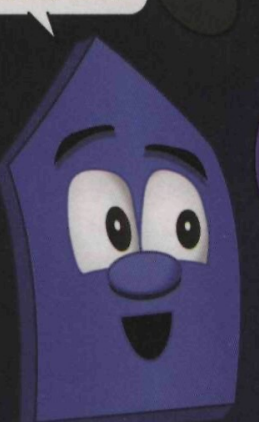

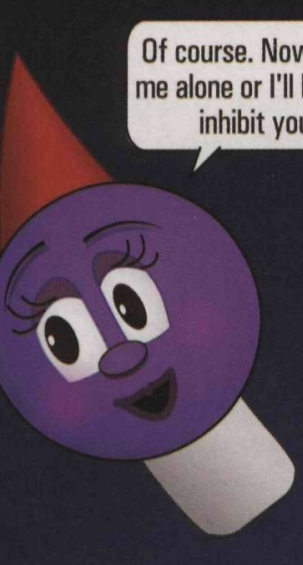
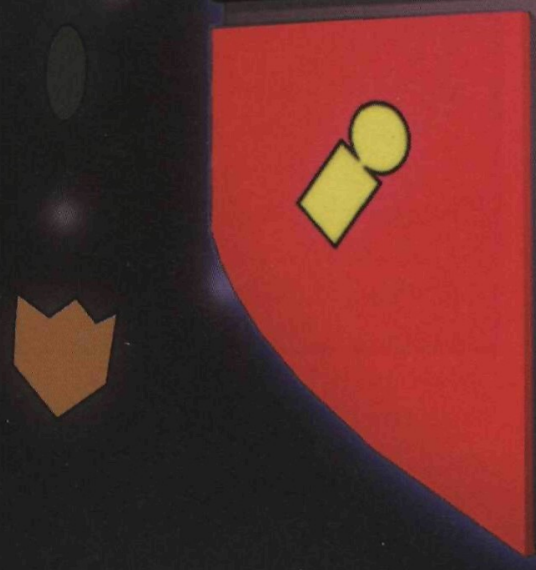
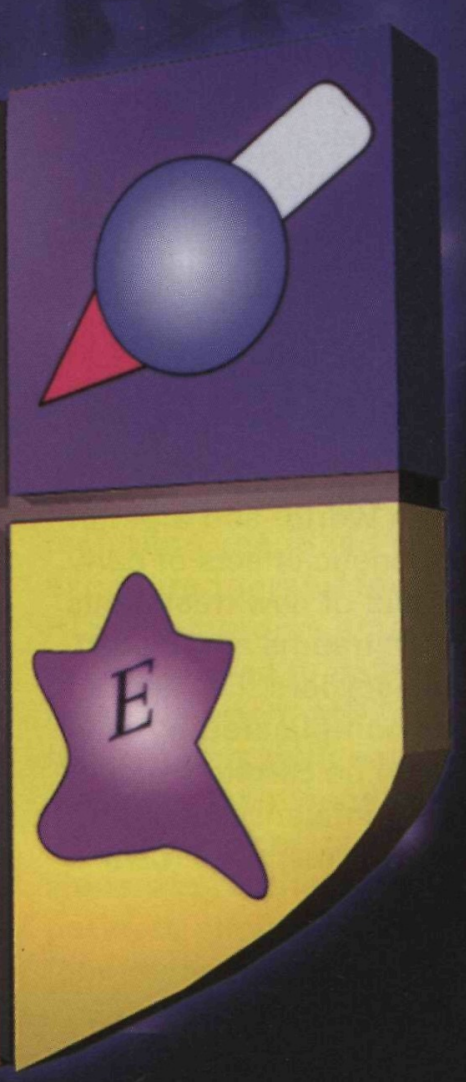

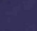

